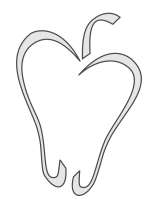

ISSN $1852-4249$

\title{
Fluorine chemistry at extreme conditions: Possible synthesis of $\mathrm{HgF}_{4}$
}

\author{
Michael Pravica, ${ }^{1 *}$ Sarah Schyck,${ }^{1}$ Blake Harris,${ }^{1}$ Petrika Cifligu, ${ }^{1}$ \\ Eunja Kim, ${ }^{1}$ Brant Billinghurst ${ }^{2}$
}

\begin{abstract}
By irradiating a pressurized mixture of a fluorine-bearing compound $\left(\mathrm{XeF}_{2}\right)$ and $\mathrm{HgF}_{2}$ with synchrotron hard x-rays $(>7 \mathrm{keV})$ inside a diamond anvil cell, we have observed dramatic changes in the far-infrared spectrum within the 30-35 GPa pressure range which suggest that we may have formed $\mathrm{HgF}_{4}$ in the following way: $\mathrm{XeF}_{2} \stackrel{h v}{\longrightarrow} \mathrm{Xe}+\mathrm{F}_{2}$ (photochemically) and $\mathrm{HgF}_{2}+\mathrm{F}_{2} \rightarrow \mathrm{HgF}_{4}(30 \mathrm{GPa}<P<35 \mathrm{GPa})$. This lends credence to recent theoretical calculations by Botana et al. that suggest that $\mathrm{Hg}$ may behave as a transition metal at high pressure in an environment with an excess of molecular fluorine. The spectral changes were observed to be reversible during pressure cycling above and below the above mentioned pressure range until a certain point when we suspect that molecular fluorine diffused out of the sample at lower pressure. Upon pressure release, $\mathrm{HgF}_{2}$ and trace $\mathrm{XeF}_{2}$ were observed to be remaining in the sample chamber suggesting that much of the $\mathrm{Xe}$ and $\mathrm{F}_{2}$ diffused and leaked out from the sample chamber.
\end{abstract}

\section{Introduction}

Mercury and cesium have been predicted to behave as a transition metal $[1,2]$ and p-block element respectively $[2,3]$ at high pressure (within the 1 Mbar range) in the presence of fluorine and thus have higher oxidation states enabling sharing/transfer of electrons from the inner shells (i.e. below the valence levels) of the elements as fluorine atoms are brought closer to the metals via high pressure. As the most electronegative element, there are a number of challenges associated with loading highly reactive and toxic molecular fluorine into a diamond anvil cell which is likely the primary reason why

\footnotetext{
*E-mail: pravica@physics.unlv.edu

1 High Pressure Science and Engineering Center (HiPSEC) and Department of Physics, University of Nevada Las Vegas (UNLV), 89154-4002 Las Vegas, Nevada, USA.

2 Far-IR beamline, Canadian Light Source, 44 Innovation Blvd, Saskatoon, SK S7N 2V3, Canada.
}

there was only one published study of the material at high pressure ( $>1 \mathrm{GPa})$ to the best of our knowledge [4]. In an effort to develop fluorine chemistry at extreme conditions, we have utilized hard x-ray induced photochemistry [5] to release molecular fluorine in situ inside a sealed and pressurized diamond anvil cell by irradiating a relatively inert and easy-to-handle, powdered or liquid (and thus easy to load) fluorine-bearing compound such as perfluorohexane $\left(\mathrm{C}_{6} \mathrm{~F}_{14}\right)[6]$, potassium tetrafluoroborate $\left(\mathrm{KBF}_{4}\right)$ [7] or $\mathrm{XeF}_{2}$. The fluorine-bearing compound is then irradiated with $\mathrm{x}$-rays that are of sufficient energy to penetrate the confining diamonds (or surrounding gasket) [7] which are typically in the hard $\mathrm{x}$-ray range $(>7 \mathrm{keV})$. As long as we are at a pressure above the solidification pressure of fluorine (2 GPa), the released atomic or molecular fluorine from irradiation is now confined in the sample hole and thus available for chemical reaction.

In the present study, we sought to verify the 
Papers in Physics, vol. 11, ART. 110001 (2019) / M. Pravica et al.

predictions of transition metal behavior of $\mathrm{Hg}$ by mixing a fluorine-bearing compound $\left(\mathrm{XeF}_{2}\right)$ with $\mathrm{HgF}_{2}$. Fluorine would be produced via x-ray irradiation of $\mathrm{XeF}_{2}$ via the following photochemical reaction:

$$
\mathrm{XeF}_{2} \stackrel{h v}{\longrightarrow} \mathrm{Xe}+\mathrm{F}_{2} .
$$

The molecular fluorine would then be available to react with $\mathrm{HgF}_{2}$ in the following way:

$$
\mathrm{HgF}_{2}+\mathrm{F}_{2} \rightarrow \mathrm{HgF}_{4}
$$

under high pressure. The goal of this effort, then, was to ascertain if any molecular changes occurred after irradiation and then after further pressurization. As our samples are typically very fluorescent after irradiation, we chose infrared spectroscopy as the means to interrogate bonding changes within our sample. As the confined sample was $\sim 3$ nano liters, we used a bright synchrotron hard x-ray source and synchrotron infrared source to produce fluorine in situ and to spectroscopically investigate our post-irradiated sample respectively.

\section{Experimental}

Due to the high reactivity of both $\mathrm{HgF}_{2}$ and $\mathrm{XeF}_{2}$ with air and water, loading of the sample was performed inside a Ar-backfilled glovebox located at the High Pressure Collaborative Access Team's sample preparation facility at the Advanced Photon Source of Argonne National Laboratory. A rhenium gasket was preindented to $20 \mu \mathrm{m}$ thickness (from $250 \mu \mathrm{m}$ initial thickness) using a symmetricstyle Diamond Anvil Cell (DAC) with diamonds that each had a culet diameter of $\sim 300 \mu \mathrm{m}$ and were IR-transmitting type I quality. A sample hole of diameter $\sim 80 \mu \mathrm{m}$ was laser drilled in the gasket [8]. Powdered xenon difluoride (Sigma Aldrich $>99 \%$ ) was pulverized with $\mathrm{HgF}_{2}$ (Sigma Aldrich $>99 \%$ ) in a 50/50 mixture by volume and was loaded via spatula into the gasket hole. One thermally-relieved ruby (for pressure measurement) was introduced into the sample which was pressurized to $10 \mathrm{GPa}$. No pressure-transmitting medium was used in our experiments and all were performed at room temperature. Raman spectroscopy was performed on the sample to verify that $\mathrm{XeF}_{2}$ was present in the loaded and pressurized sample.
The loaded sample was then irradiated with "white" x-rays produced at the 16 BM-B beamline at the Advanced Photon Source (APS). The beam was $\sim 30$ microns in diameter. The $\mathrm{HgF}_{2}$ and $\mathrm{XeF}_{2}$ mixture was irradiated for more than five hours at pressures above $10 \mathrm{GPa}$ to avoid any material losses triggered by the X-ray induced decomposition of $\mathrm{XeF}_{2}$. XRD patterns of the sample were taken at the 16 ID-B using monochromatic x-rays that were collected by a MAR345® image plate detector. We also note that no irradiation-induced changes in pure $\mathrm{HgF}_{2}$ were observed at any pressure in separate experiments. Thus, only $\mathrm{XeF}_{2}$ is photochemically-affected by x-rays.

The irradiated sample was then transported to the 02B1-1 far-infrared (far-IR) beamline of the Canadian Light Source (CLS) where IR Spectroscopy measurements at various pressures were carried out in situ inside the DAC. Pressure was measured using a homemade ruby-fluorimeter constructed by our group located on site at the CLS. The IR collection system consisted of a plexiglass enclosure housing the DAC and collection optics which was in front of the Fourier Transform-IR system and was continuously purged from water vapor (measured by a humidity sensor) using positive pressure nitrogen gas blowoff from a liquid nitrogen dewar. A horizontal microscope system collected far-IR spectra. The IR beam was redirected from the sample compartment of a Bruker IFS $125 \mathrm{HR}(\mathrm{R})$ spectrometer to within the working distance of a Schwarzchild objective which focused IR light onto the sample. A similar light focusing objective placed behind the sample was used to collect the transmitted light, directing it onto an offaxis parabolic mirror which refocused the IR light into an Infrared Laboratories® Si bolometer. The spectrometer was equipped with a 6-micron mylar beamsplitter. The data was collected using a scanner velocity of $40 \mathrm{kHz}, 12.5-\mathrm{mm}$ entrance aperture, with a $1 \mathrm{~cm}^{-1}$ resolution. The Si bolometer was set for a gain of $16 \times$. Interferograms were transformed using a zero filling factor of 8 and a 3-term Blackman Harris apodization function.

FT-IR spectral scans typically required 15 minutes to acquire and all measurements were performed at room temperature. 
Papers in Physics, vol. 11, ART. 110001 (2019) / M. Pravica et al.

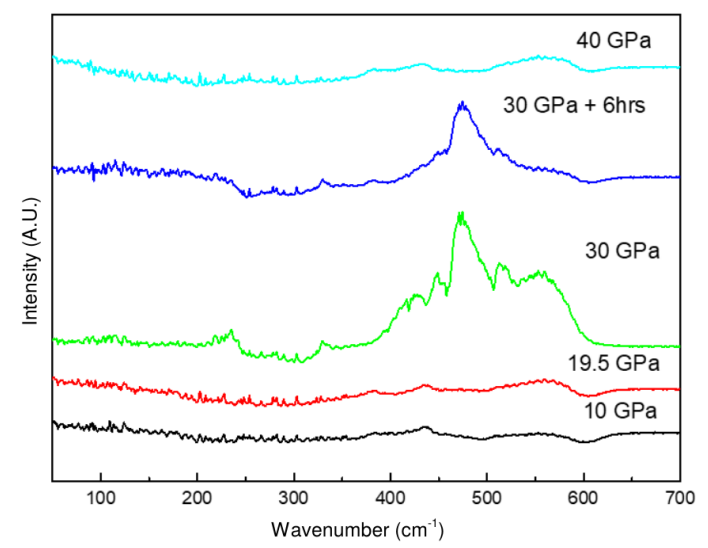

Figure 1: Transmission far-IR spectra of $\mathrm{HgF}_{2}$ and $\mathrm{XeF}_{2}$ mixture pressurized up to $30 \mathrm{GPa}$ and held at $30 \mathrm{GPa}$ for 6 hours then pressurized to $40 \mathrm{GPa}$. As the pressure is increased beyond $19.5 \mathrm{GPa}$, a broad multiplet of spectral lines appear near $474 \mathrm{~cm}^{-1}$ and one smaller mode appears near $234 \mathrm{~cm}^{-1}$. The patterns disappear in the $40 \mathrm{GPa}$ spectra.

\section{Results}

After initial loading at the APS, the sample possessed a greenish yellow tint demonstrating the presence of $\mathrm{HgF}_{2}$. After further pressurization at the CLS, the sample significantly darkened. We present our IR spectral data in the $35-650 \mathrm{~cm}^{-1}$ range in Fig. 1. We first compressed the sample from $10 \mathrm{GPa}$ up to $40 \mathrm{GPa}$ recording spectral patterns along the way. As is evident from the figure, a peak near $235 \mathrm{~cm}^{-1}$ and a multiplet of peaks centered near $474 \mathrm{~cm}^{-1}$ appear around $30 \mathrm{GPa}$. We allowed the sample to remain at $30 \mathrm{GPa}$ for 6 hours and then took another IR spectrum to examine stability of the new peaks with time. The $235 \mathrm{~cm}^{-1}$ peak vanished or was severely diminished within the signal to noise of our system and the multiplet centered around $474 \mathrm{~cm}^{-1}$ largely disappeared or severely diminished with the exception of the peak itself. Upon further pressurization to $40 \mathrm{GPa}$, the highest pressure we subjected the sample to, the patterns completely disappear.

The sample pressure was then reduced to $35 \mathrm{GPa}$ (Fig. 2, curve a) to ascertain if the observed peaks returned which they did as evidenced in the $32 \mathrm{GPa}$ pattern in Fig. 2, curve b. Pressure was again increased to $35 \mathrm{GPa}$ and the pattern again disap-

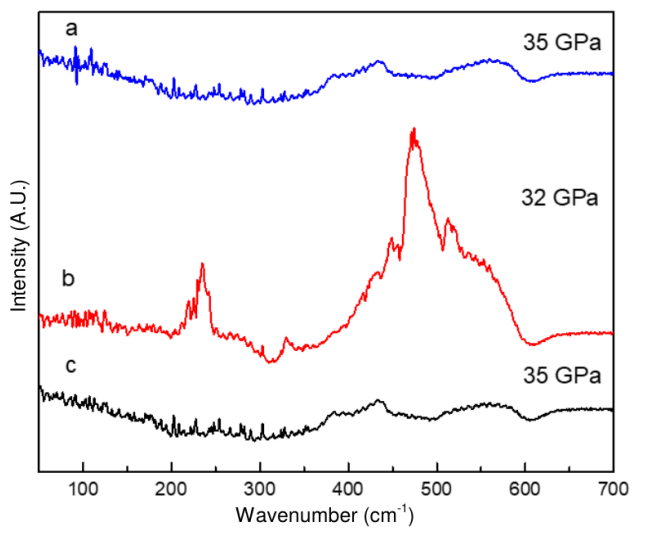

Figure 2: Transmission far IR spectra of the irradiated $\mathrm{XeF}_{2}$ and $\mathrm{HgF}_{2}$ mixture as the sample was decompressed from $35 \mathrm{GPa}$ (trace a) to $32 \mathrm{GPa}$ (trace b) and then recompressed to $35 \mathrm{GPa}$ (trace c) demonstrating reversibility of the peak structure at $32 \mathrm{GPa}$. This pressure-cycled sequence occurred after the first viewing of the feature around $30 \mathrm{GPa}$ present in Fig. 1.

peared (Fig. 2, curve c). Pressure was reduced to just above ambient $(\sim 1 \mathrm{GPa})$ and the sample returned to its original white/yellow appearance before irradiation (white). Figure 3 displays photos of the sample at various stages. Raman spectroscopy was performed upon returning the sample to the Pravica Raman facility at UNLV indicating that only $\mathrm{HgF}_{2}$ and a residual amount of $\mathrm{XeF}_{2}$ remained in the sample chamber. X-ray diffraction (XRD) patterns taken of the sample before irradiation and after irradiation, compression and decompression to ambient conditions (see Fig. 4) further verifies the claim that the $\mathrm{Xe}$ and $\mathrm{F}_{2}$ (produced via irradiation of $\mathrm{XeF}_{2}$ ) leaked out from the gasket once the pressure was reduced to near ambient conditions. There is no indication that the rhenium gasket suffered any significant chemical reaction from the $\mathrm{F}_{2}$ (see Fig. 4). We have observed this behavior of little or no diffusion of $\mathrm{F}_{2}$ in our samples in prior experiments that produced $\mathrm{F}_{2}$ from $\mathrm{KBF}_{4}$ leading to little or no gasket damage [10] and no discernible reaction with the diamonds [4]. 
Papers in Physics, vol. 11, ART. 110001 (2019) / M. Pravica et al.

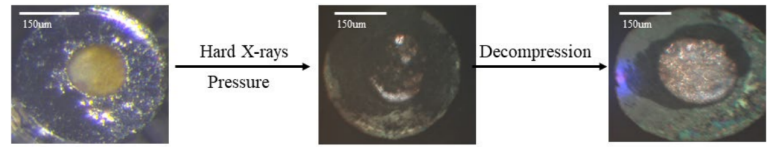

Figure 3: Progression sequence of the sample. The first photo on the left represents the mixed $\mathrm{XeF}_{2}+\mathrm{HgF}_{2}$ sample near $10 \mathrm{GPa}$ after sample loading. A yellowish hue is evident due to the presence of $\mathrm{HgF}_{2}$. The second (middle) photo illustrates darkening of the sample after irradiation and pressurization to $25 \mathrm{GPa}$ and persisted in this visual state until $40 \mathrm{GPa}$, the highest pressure in this study. The final photo on the right demonstrates that the sample has returned to its original appearance after reducing pressure to ambient conditions.

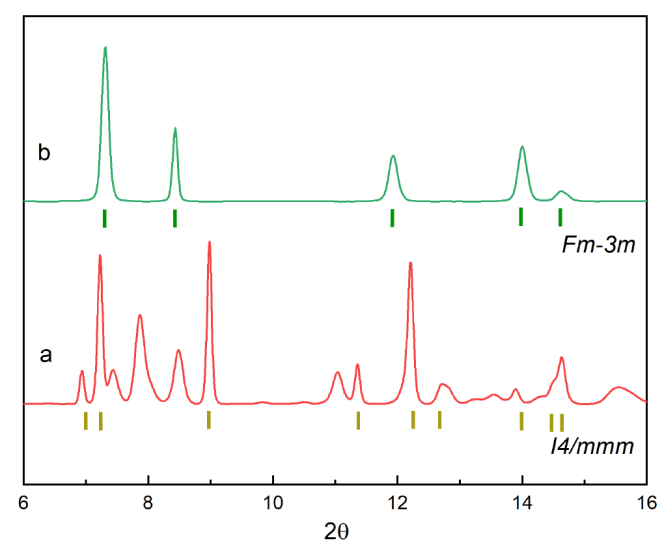

Figure 4: $\mathrm{XRD}$ patterns of the $\mathrm{HgF}_{2} / \mathrm{XeF}_{2}$ mixture (a) before x-ray irradiation and (b) after irradiation, pressurization, and decompression indicating that the $\mathrm{XeF}_{2}$ leaked out from the gasket in the form of $\mathrm{Xe}$ and $\mathrm{F}_{2}$ (produced from the initial x-ray irradiation) leaving only $\mathrm{HgF}_{2}$ in the sample chamber in the Fm-3m crystalline structure. The vertical olive green bars in (a) represent the tetragonal crystal structure of $\mathrm{XeF}_{2}$ with the $\mathrm{I} 4 / \mathrm{mmm}$ space group [11].

\section{Discussion}

$\mathrm{HgF}_{4}$ in the gaseous state has a predicted IR mode $\left(\mathrm{A}_{2 u}\right)$ near $233 \mathrm{~cm}^{-1}$ [9] which agrees well with the mode we observed near $235 \mathrm{~cm}^{-1}$ though we recognize that our mode was observed in the solid state (not the gaseous state) and is at very high pressure. We suspect that the feature near $474 \mathrm{~cm}^{-1}$ is an overtone of the mode near $235 \mathrm{~cm}^{-1}$. Botana et al. have calculated stability of $\mathrm{HgF}_{4}$ in the 38-73
GPa pressure range; that $\mathrm{HgF}_{3}$ and $\mathrm{HgF}_{4}$ are both stable from 73-200 GPa; and that from 200-500 $\mathrm{GPa}$, only $\mathrm{HgF}_{3}$ is the stable compound with $\mathrm{Hg}$ in the +3 oxidation state [1]. Seeking to confirm this prediction, we pressurized the DAC into the $30 \mathrm{GPa}$ and higher pressure range. As is apparent from our data, a new compound with mercury appears to form near $30 \mathrm{GPa}$ and then disappears around $35 \mathrm{GPa}$. The compound forms reversibly with pressure cycling. Upon further reduction of pressure to ambient conditions, the sample turned white (as it was originally before being irradiated). Raman spectroscopy confirmed only the presence of $\mathrm{HgF}_{2}$ indicating that the $\mathrm{Xe}$ and $\mathrm{F}_{2}$ leaked out from the gasket. The process (irradiation, pressurization and return to ambient) is visually described in Fig. 3.

We note in passing that we performed a purely high pressure mid-IR study of just the $\mathrm{XeF}_{2}+$ $\mathrm{HgF}_{2}$ mixture (see Fig. 5) and found no evidence of any significant spectral changes (with the exception of a phase transition near $5 \mathrm{GPa}$ from $\mathrm{HgF}_{2}$ ) demonstrating that $\mathrm{x}$-ray irradiation in combination with high pressure is necessary to produce the interesting features observed in Fig. 2.

\section{Conclusions}

We have performed a synchrotron far-IR experiment on an irradiated mixture of $\mathrm{XeF}_{2}$ and $\mathrm{HgF}_{2}$ pressurized in a DAC. The irradiation was performed to release molecular fluorine inside the sample chamber at high pressure in situ thereby obviating the need to load toxic and reactive molecular fluorine inside the diamond cell. Upon further pressurization just above $30 \mathrm{GPa}$, we observed the dramatic appearance of a peak or peaks centered near $234 \mathrm{~cm}^{-1}$ and likely an overtone near $474 \mathrm{~cm}^{-1}$ in a narrow pressure range somewhere between $30-35$ GPa which appears to be reversible and which appears to correlate with the calculated $\mathrm{A}_{2 u}$ mode of $\mathrm{HgF}_{4}$. Our observation differs somewhat from the predictions of Botana et al. of a 38-73 GPa pressure range of stability [1] but given the challenges associated with connecting theory and experiment at high pressure and given the complex chemistry occurring during and after hard x-ray irradiation and at high pressures, our results are nevertheless encouraging. 
Papers in Physics, vol. 11, ART. 110001 (2019) / M. Pravica et al.

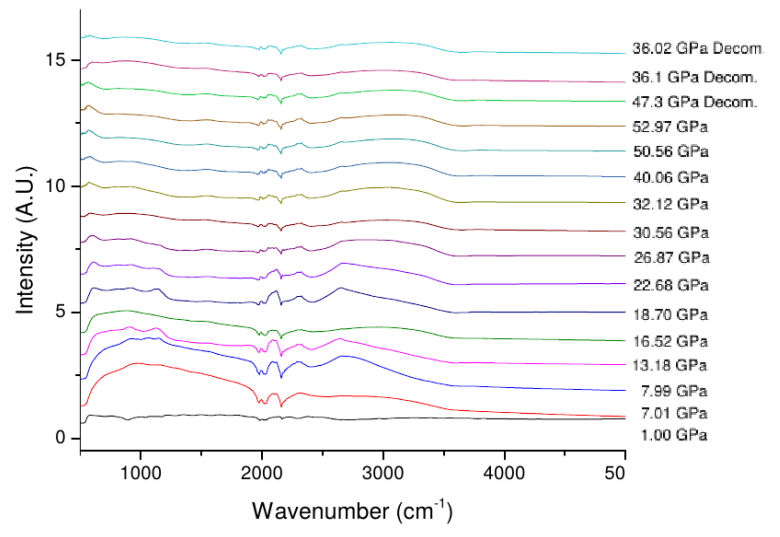

Figure 5: Transmission mid-IR spectra of $\mathrm{HgF}_{2}$ and $\mathrm{XeF}_{2}$ non-irradiated mixture pressurized up to $36 \mathrm{GPa}$.

Upon release of pressure to ambient, the fluorine and $\mathrm{Xe}$ produced by the irradiation of $\mathrm{XeF}_{2}$ likely leaked out and $\mathrm{HgF}_{2}$ remained inside along with residual $\mathrm{XeF}_{2}$. Though far-IR experiments do not by themselves prove the formation of $\mathrm{HgF}_{4}$, we are nevertheless encouraged by our results. Further experiments are planned to confirm and further verify our results. We anticipate that this seminal experiment will further encourage development of fluorine chemistry at extreme conditions.

Acknowledgements - We thank Tim May and Zhenxian Liu for help in the far-IR and midIR measurements, respectively. We gratefully acknowledge support from the Department of Energy National Nuclear Security Administration (DOENNSA) under Award Number DE-NA0002912. We also acknowledge support from the DOE Cooperative Agreement No. DE-FC08-01NV14049 with the University of Nevada, Las Vegas. Portions of this work were performed at HPCAT (Sector 16), Advanced Photon Source (APS), Argonne National Laboratory. HPCAT operations are supported by DOE-NNSA under Award No. DE-NA0001974 and DOE-BES under Award No. DE-FG0299ER45775, with partial instrumentation funding by NSF. APS is supported by DOE-BES, under Contract No. DE-AC02-06CH11357. A portion of the research described in this paper was performed at the far-IR beamline of the Canadian Light
Source, which is supported by the Natural Sciences and Engineering Research Council of Canada, the National Research Council Canada, the Canadian Institutes of Health Research, the Province of Saskatchewan, Western Economic Diversification Canada, and the University of Saskatchewan.

[1] J Botana, X Wang, C Hou, D Yan, H Lin, Y Ma, MS Miao, Mercury under pressure acts as a transition metal: Calculated from first principles, Angew. Chem. Int. Ed. Engl. 54, 9280 (2015).

[2] MS Miao, J Botana, M Pravica, D Sneed, C Park, Inner-shell chemistry under high pressure, Jap. J. Appl. Phys. 56, 05FA10 (2017).

[3] MS Miao, Caesium in high oxidation states and as a p-block element, Nature Chem. 5, 846 (2013).

[4] D Schiferl, S Kinkead, R Hanson, D Pinnick, Raman spectra and phase diagram of fluorine at pressures up to $6 \mathrm{GPa}$ and temperatures between 10 and $320 \mathrm{~K}$, J. Chem. Phys. 87, 3016 (1987).

[5] M Pravica, L Bai, C Park, Y Liu, M Galley, J Robinson, N Bhattacharya, Note: A novel method for in situ loading of gases via $x$-ray induced chemistry, Rev. Sci. Instrum. 82, 106102 (2011).

[6] M Pravica, D Sneed, M White, Y Wang, Note: Loading method of molecular fluorine using $x$ ray induced chemistry, Rev. Sci. Instrum. 85, 086110 (2014).

[7] M Pravica, M White, Y Wang, Y Xiao, $\mathrm{P}$ Chow, Hard $x$-ray induced synthesis of $\mathrm{OF}_{2}$, Chim. Oggi 36, 50 (2018).

[8] R Hrubiak, S Sinogeikin, E Rod, G Shen, The laser micro-machining system for diamond anvil cell experiments and general precision machining applications at the High Pressure Collaborative Access Team, Rev. Sci. Instrum. 86, 072202 (2015).

[9] M Kaupp, HG von Schnering, Gaseous Mercury (IV) Fluoride, $\mathrm{HgF}_{4}$ : An ab initio study, Angew. Chem. Int. Ed. Engl. 32, 861 (1993). 
Papers in Physics, vol. 11, ARt. 110001 (2019) / M. Pravica et al.

[10] M Pravica, M White, Y Wang, A novel method for generating molecular mixtures at extreme conditions: The case of Fluorine and Oxygen, AIP Conf. Proc. 1793, 060030 (2017).
[11] G Wu, X Huang, Y Huang, L Pan, F Li, X Li, M Liu, B Liu, T Cui, Confirmation of the structural phase transitions in $\mathrm{XeF}_{2}$ under high pressure, J. Phys. Chem. C. 121, 6264 (2017). 